\title{
Targeted Fluorogenic Cyanine Carbamates Enable In Vivo Analysis of Antibody-Drug Conjugate Linker Chemistry
}

\author{
Syed Muhammad Usama, ${ }^{1}$ Sierra C. Marker, ${ }^{1}$ Donald R. Caldwell, ${ }^{1}$ Nimit L. Patel, ${ }^{2}$ Yang Feng, ${ }^{3}$ Joseph D. Kalen 2 , Brad St. \\ Croix, ${ }^{3}$ and Martin Schnermann ${ }^{1 *}$ \\ ${ }^{1}$ Chemical Biology Laboratory, Center for Cancer Research, National Cancer Institute, Frederick, Maryland 21702, United \\ States
}

${ }^{2}$ Small Animal Imaging Program, Frederick National Laboratory for Cancer Research, Leidos Biomedical Research Inc., Frederick, Maryland 21702, United States

${ }^{3}$ Tumor Angiogenesis Unit, Mouse Cancer Genetics Program (MCGP), National Cancer Institute (NCI), NIH, Frederick, Maryland, United States

KEYWORDS antibody drug conjugate, fluorogenic probes, near infrared optical imaging, linker chemistry, protease

\begin{abstract}
Antibody-drug conjugates (ADCs) are a rapidly emerging therapeutic platform. The chemical linker between the antibody and the drug payload plays an essential role in the efficacy and tolerability of these agents. New methods that quantitively assess cleavage efficiency in complex tissue settings could provide valuable insights into the ADC design process. Here we report the development of a near-infrared (NIR) optical imaging approach that measures the site and extent of linker cleavage in mouse models. This approach is enabled by a superior variant of our recently devised cyanine carbamate (CyBam) platform. We identify a novel tertiary amine-containing norcyanine, the product of CyBam cleavage, that exhibits dramatically increased cellular signal due to improved cellular permeability and lysosomal accumulation. The resulting cyanine lysosome-targeting carbamates (CyLBams) are $\sim 50 \mathrm{X}$ brighter in cells, and we find this strategy is essential for high-contrast in vivo targeted imaging. Finally, we compare a panel of several common ADC linkers across two antibodies and tumor models. These studies indicate that cathepsin-cleavable linkers provide dramatically higher tumor activation relative to hindered or non-hindered disulfides - an observation that is only apparent with in vivo imaging. This strategy enables quantitative comparisons of cleavable linker chemistries in complex tissue settings with implications across the drug delivery landscape.
\end{abstract}

\section{INTRODUCTION}

Antibody drug conjugates (ADCs) combine the specificity of monoclonal antibodies (mAbs) and the potency of small molecule therapeutics. To date, 10 ADCs have received FDA approval and many others $(>60)$ are in clinical trials. ${ }^{1,2}$ ADC activity generally requires lysosomal processing of a linker domain to release the active payload. Consequently, the linker component should be stable in circulation, but selectively cleaved following target binding and internalization - a significant chemical challenge. ${ }^{3-7}$

To assess the state-of-the-art and to guide future linker discovery efforts, a means to quantitively compare ADC linker chemistry in vivo would be of significant utility. ADCs are conventionally assessed by examining tumoricidal activity and toxicity profiling. While these methods are important benchmarks, they provide only indirect insights into the site and mechanism of drug release. ${ }^{8-10}$ Enzyme-linked immunosorbent assays (ELISAs) are also broadly employed, but only determine the blood-pool concentration and biodistribution of the antibody component. ${ }^{11-12}$ Radiolabeling methods can provide important insights regarding $\mathrm{mAb}$ localization, but are costly and do not directly report on the linker cleavage step. ${ }^{13-16}$
Optical imaging has the potential to provide critical insights to the ADC design and optimization process. Prior efforts using stimuli-responsive fluorophores with conventional visible wavelengths have quantified payload processing and internalization kinetics in cellular imaging experiments. ${ }^{17-19}$ However, these probes are not suitable for applications in deep tissue due to the poor penetration depth of wavelengths in the visible region. Complementing these efforts, approaches using alwaysON near-infrared (NIR) probes have provided insights into tumor and off-target uptake ${ }^{20-23}$ Fluorogenic turn-ON probes that use NIR wavelengths ( 700 to $900 \mathrm{~nm}$ ) have significant potential to provide insight into dynamics and localization of biological phenomena in complex tissue settings. We recently developed the first class of NIR fluorogenic probes based on the heptamethine cyanine core. ${ }^{24}$ These fluorogenic cyanine carbamates (CyBams) exhibit exceptional turn-ON ratios and untargeted variants enabled in vivo imaging in a metastatic tumor model. We hypothesized $\mathrm{mAb}$-conjugated variants could provide a real time, quantitative approach to determine the site and extent of ADC linker cleavage in complex model organisms. 
Here we detail the development of the first activatable mAb targeted probes with absorbance and emission maxima beyond $700 \mathrm{~nm}$. To obtain sufficient signal for in vivo imaging, we optimized the cellular uptake and retention of the norcyanine scaffold - the released fluorescent product of linker cleavage. These efforts reveal the installation of a basic amine into the probe dramatically improves cellular photon output, likely due to enhanced lysosomal uptake and retention. Converting the optimized norcyanine into the corresponding cyanine lysosome-targeting carbamate (CyLBam) dramatically improves the in vitro signal and is essential for high-contrast in vivo optical imaging of $\mathrm{mAb}$ conjugates. Finally, we test a panel of commonly used ADC linkers in two tumor models. We find cathepsin-cleavable linkers outperform reductively cleaved disulfide linkers with dramatic differences in tumor uptake - a distinction that is only apparent with in vivo imaging. Broadly, this approach provides a general means to assess cleavable linker efficiency and specificity, with applications from the cellular to organismal scales.

\section{RESULTS AND DISCUSSION}

\section{Optimization of Norcyanine Signal}

Prior to pursuing in vivo imaging of $\mathrm{mAb}$ conjugates, we set out to improve the cellular signal of the product of CyBam activation, the pH-sensitive norcyanine. While the previously reported sulfonated norcyanine, Sulfo-NorCy7 (Figure 1A), can provide high contrast imaging, relatively high concentrations $(20-40 \mu \mathrm{M})$ are required for sufficient in vitro signal. ${ }^{24-27} \mathrm{We}$ anticipated this requirement might be problematic for $\mathrm{mAb}$ targeted imaging because probe concentrations are intrinsically limited by antigen levels. Of note, microscopy studies indicate that the cellular signal of Sulfo-NorCy7 is predominately from the lysosome - an observation consistent probe protonation and a $\mathrm{p} K_{\mathrm{a}}$ between 4 and $5 .{ }^{24} \mathrm{We}$ therefore presume poor lysosomal accumulation of the charged sulfonated variants is responsible for the modest cellular fluorescence of this probe. We hypothesized that improving cellular permeability and, perhaps, introducing a lysosomal targeting element could improve the fluorescent output.

To approach this problem, we designed and synthesized a small panel of chemically diverse norcyanines. These compounds contain a C4'-phenyl-substituent appended to the polymethine chromophore, which we and others have found to improve synthetic accessibility, photostability, and serum stability. ${ }^{28-33}$ To test the question of cell permeability alone, we included two hydrophobic derivatives, OMe-NorCy7 and H-Nor-Cy7, (Figure 1A). Commonly used lysotracker probes contain tertiary amines, suggesting this functional group can promote lysosomal targeting. ${ }^{34-36}$ To test if this might apply to norcyanines, we prepared indolenine-substituted sulfonamide derivatives modified with both the hydrophobic piperidine (Pip-NorCy7) and the tertiary amine-containing $N$-Me piperazine $(N$-Me-PipNorCy7) substituents. We also prepared another "lysotrackerlike" derivative, $\boldsymbol{N}$-Me-NorCy7, in which the central ring system contains an $N$-methyl substituent. These novel norcyanines were synthesized in 3-4 steps, as described in the supporting information.

With access to this panel of probes, we investigated their photophysical properties and cellular uptake. All six probes exhibit absorbance and emission maxima above $700 \mathrm{~nm}$, similar extinction coefficients and $\mathrm{p} K_{\mathrm{a}}$ 's between 4.4 and 5.2 (Figure 1B,C

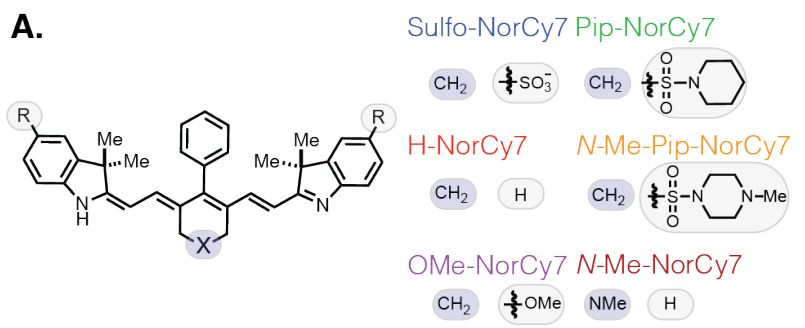

B.

C.
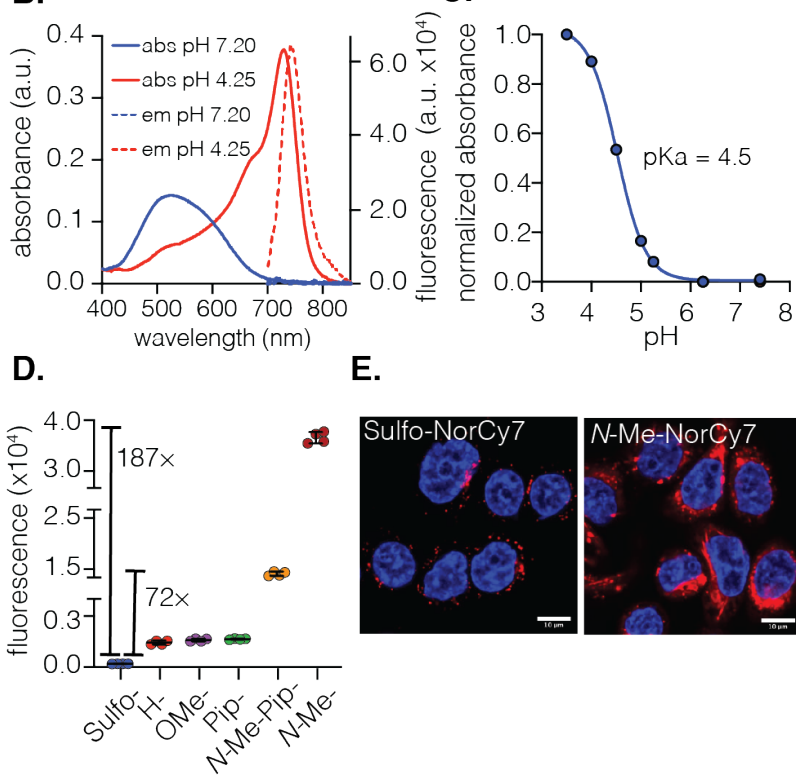

Figure 1. A. Structures of norcyanines Sulfo-, H-, OMe-, Pip-, $\mathrm{N}$-Me-Pip- and $\mathrm{N}$-Me-NorCy7. B. Absorbance and fluorescence spectra w/ $690 \mathrm{~nm}$ excitation of $\boldsymbol{N}$-Me-NorCy7 $(5 \mu \mathrm{M})$ in PBS pH 7.20 and acetate buffer $\mathrm{pH} 4.25$. C. Determination of $\mathrm{p} K_{\mathrm{a}}(10 \mu \mathrm{M})$ in buffers ranging from $\mathrm{pH} 3.5$ to $\mathrm{pH}$ 7.4. D. Flow cytometry quantification of in vitro uptake of norcyanine heptamethine cyanine $(5 \mu \mathrm{M})$ in MDA-MB-468 after $1 \mathrm{~h}$ incubation. Geometric mean fluorescent intensity $( \pm \mathrm{SD})$ of fluorescent signal in the cells is shown $(n=4$ independent experiments; $\sim 10,000$ cells counted). E. Confocal fluorescent images (63X) of $\mathrm{N}$-MeNorCy7 and Sulfo-NorCy7 $(10 \mu \mathrm{M})$ after $6 \mathrm{~h}$ incubation with MDA-MB-468. Fluorescent signal from the probe and nucleus (Hoechst) is pseudo-colored red and blue, respectively.

for $\mathbf{N}$-Me-NorCy7, Table 1, Figure S1). We first established that the NorCy7 series had minimal toxicity in MDA-MB-468 and MCF-7 cells (Figure S2). Next, we quantified signal in MDA-MB-468 cells at 1,3 and $6 \mathrm{~h}$ time points with flow

\begin{tabular}{|c|c|c|c|}
\hline & $\begin{array}{c}\lambda_{\max a b s} / \lambda_{\text {max em }}(\mathrm{nm}) \\
\end{array}$ & $\begin{array}{c}\varepsilon_{\max } \\
\left(\mathrm{M}^{-1} \mathrm{~cm}^{-1}\right)\end{array}$ & $\mathrm{p} K_{\mathrm{a}}$ \\
\hline Sulfo-NorCy $7^{\mathrm{a}}$ & $755 / 775$ & 73,500 & 5.2 \\
\hline $\mathrm{H}-$ NorCy $7^{\mathrm{b}}$ & $750 / 770$ & 63,900 & 5.8 \\
\hline OMe-NorCy $7^{\mathrm{b}}$ & $775 / 790$ & 56,800 & 5.5 \\
\hline Pip-NorCy $7^{\circ}$ & $760 / 780$ & 17,200 & 4.4 \\
\hline N-Me-Pip-NorCy $7^{\text {a }}$ & a $760 / 775$ & 45,000 & 4.5 \\
\hline N-Me-NorCy $7^{a}$ & $730 / 750$ & 57,600 & 4.6 \\
\hline
\end{tabular}

Table 1. Summary of photophysical properties $\left(\lambda_{\max a b s}, \lambda_{\max }\right.$ em and $\left.\varepsilon_{\max }\right)$ of NorCy 7 series of compounds. ${ }^{a}$ acetate buffer (pH 4.5); ${ }^{b} \mathrm{MeOH}$ acetate buffer $\mathrm{pH} 4.5$ (2:1); ${ }^{\mathrm{c}} \mathrm{MeOH}$ :acetate buffer $\mathrm{pH} 3.75$ (2:1). 
cytometry using the APC-Cy7 channel. At all three time points, the uptake of the six norcyanines follows the rank order: $\mathrm{N}$-MeNorCy7 > N-Me-Pip-NorCy7 $>$ Pip-NorCy7, OMeNorCy7, H-Nor-Cy7 >> Sulfo-NorCy7 (Figure 1D and S3, S31-33). To our delight, the $N$-Me-NorCy7 and $N$-Me-PipNorCy7 exhibit 187-fold and 72-fold higher uptake, respectively, in MDA-MB-468 cells after only $1 \mathrm{~h}$ incubation compared to previously used Sulfo-NorCy7. The fluorescent signal remained constant at the 1,3 and $6 \mathrm{~h}$ time points suggesting efficient uptake and high retention of tertiary amine substituted norcyanines ( $N$-Me-NorCy7 and $N$-Me-Pip-NorCy7). In contrast, the signal from Sulfo-NorCy 7 increased steadily overtime and was $\sim 3$-fold higher after $6 \mathrm{~h}$ relative to $1 \mathrm{~h}$ (Figure S3). Using confocal microscopy, we confirmed that the subcellular localization of the $\mathbf{N}$-Me-NorCy7 is lysosomal, with dramatically higher signal than SulfoNorCy7 (Figure 1E, Figure S4-5). The relatively modest signal of the three hydrophobic derivatives, Pip-NorCy7, OMe-NorCy7, and H-NorCy7, indicates that the tertiary amine, the lysotracker-mimicking feature, is essential for fluorescent signal enhancement. Overall, this data indicates that incorporation of a single basic amine dramatically enhances cellular uptake and lysosomal localization of norcyanines. The enhanced cellular signal and synthetic accessibility

A.
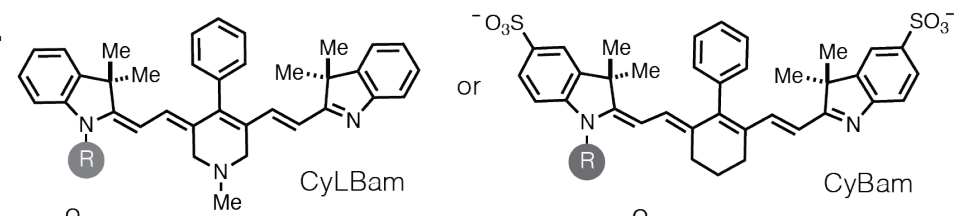

$\mathrm{R}=\mathrm{s}_{2}$

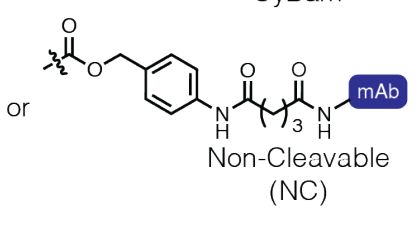

C.

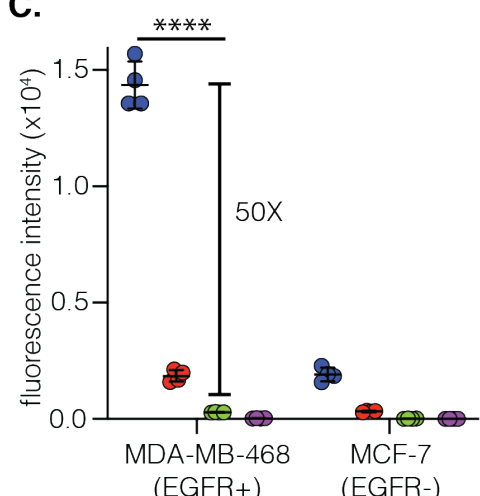

D.

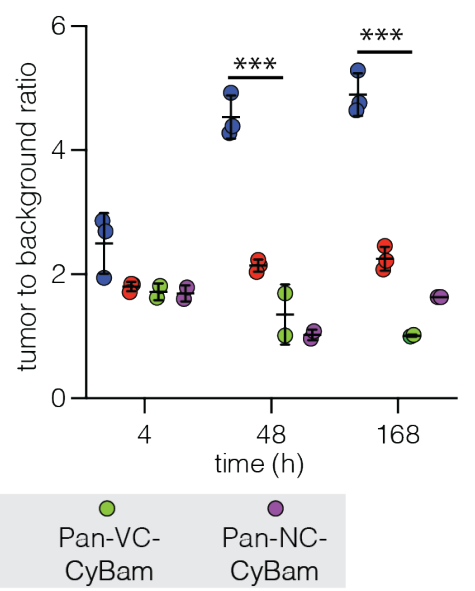

B.
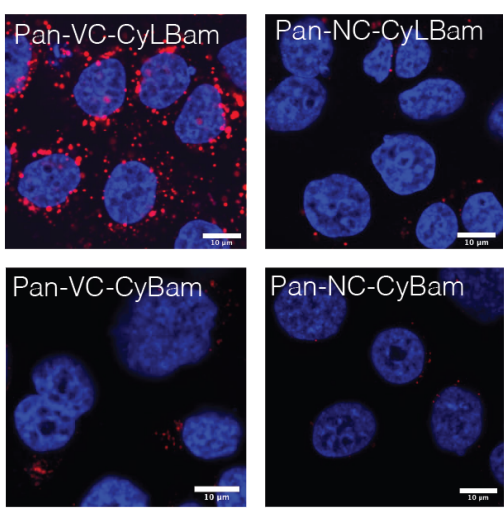

E. Pan-VC-CyLBam

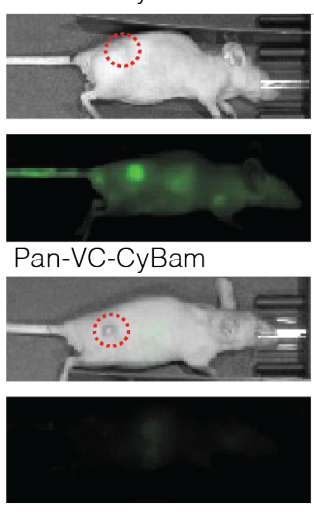

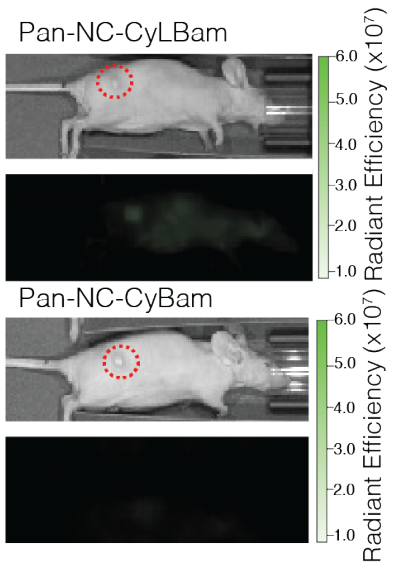

Figure 2. A. Structures of CyLBam and CyBam probes, and VC (cathepsin-cleavable val-cit) and NC (non-cleavable) linkers. B. Confocal images (63X) of Pan-VC-CyLBam, Pan-NC-CyLBam, Pan-VC-CyBam, and Pan-NC-CyBam in MDA-MB$468(\mathrm{EGFR}+)$ after $24 \mathrm{~h}$ incubation. Fluorescent signal from the probe and nucleus (Hoechst) is shown in red and blue, respectively. C. Flow Cytometry quantification of in vitro uptake in MDA-MB-468 (EGFR+) and MCF-7 (EGFR-) after 24 h incubation. All antibody conjugates were labeled at DOL 4 . Geometric mean fluorescent intensity $( \pm$ SD) of fluorescent signal in the cells is shown ( $n=4$ independent experiments; $\sim 10,000$ cells counted). D. Imaging of probes (200 $\mu \mathrm{g}$; DOL 4) intravenously injected in a xenograft model of female athymic nude mice implanted with MDA-MB-468 tumors $(n=3)$. Tumor-to-background (TBR) ratios, the fluorescent signal of the tumor relative to an equal area in the neck, at different time intervals (4, 48 and 168 h). E. IVIS fluorescent images $48 \mathrm{~h}$ post injection. Tumors are highlighted in dotted red circles. Data points are displayed as mean $\pm \mathrm{SD}$, and the p-values were evaluated by the Student's t-test (*** p-value $\leq 0.001$, **** $\mathrm{p}$-value $\leq 0.0001)$. 
We first compared the series of Pan probes in cellular assays. The four conjugates, Pan-VC-CyLBam, Pan-NC-CyLBam, Pan-VC-CyBam, Pan-NC-CyBam, (Figure 2A) were tested in MDA-MB-468 (EGFR+) and MCF-7 (EGFR-) cells. ${ }^{41-42}$ The fluorescent signal emitting from Pan-VC-CyLBam was 50fold higher than Pan-VC-CyBam after incubation with MDAMB-468 cells for $24 \mathrm{~h}$ (Figure 2C and S34-37). Validating the role of receptor-mediated uptake, the fluorescent signal of both probes was lower in MCF-7 cells. As expected, the non-cleavable probes, Pan-NC-CyLBam and Pan-NC-CyBam, did not exhibit any significant fluorescent signal in either cell line. Confocal microscopy confirmed the trends observed by flow cytometry, as well as the lysosomal uptake of the released norcyanine (Figure 2B and S6-8).

We then set out to test if the improved in vitro signal of the CyLBam conjugate would translate to high contrast animal imaging. The conjugates $(200 \mu \mathrm{g})$ were injected intravenously into female athymic nude mice with MDA-MB-468 xenograft tumors $\left(25-35 \mathrm{~mm}^{3}\right)$. The mice were imaged at 4, 24, 48, 72, and $168 \mathrm{~h}$ post-injection using an In Vivo Imaging System (IVIS) (Figure 2E and S9-14). After $24 \mathrm{~h}$, a strong fluorescent signal was observed in tumors injected with Pan-VC-CyLBam and the signal persisted over $168 \mathrm{~h}$, with tumor-to-background ratios (TBRs) between 4 and 5. In contrast, the Pan-VCCyBam group had lower TBRs (1-2) at all time points - similar to the two non-cleavable probes, Pan-NC-CyLBam and PanNC-CyBam. Significant liver signal was observed with the two probes containing the protease cleavable linker (Pan-VCCyLBam and Pan-VC-CyBam) at early time points ( $4 \mathrm{~h}$ and $24 \mathrm{~h}$ ), and the fluorescent signal decreased afterwards (Figure S15-16). The selective hepatic signal of Pan-VC-CyBam and its time-dependent disappearance suggests that the released Sulfo-NorCy7 is rapidly cleared through hepatobiliary pathways. Thus, the reduced tumor signal observed with this probe likely reflects lower tumor retention (compared to $\mathrm{N}$-MeNorCy7) and subsequent clearance. Overall, these results suggest that the improved cellular retention of $\mathrm{N}$-Me-NorCy7, first observed in vitro, extends to an in vivo setting. Critically, these observations set the stage for us to evaluate a series of ADC linkers.

\section{Quantitative Comparison of ADC Linkers}

The two most extensively studied classes of ADC linkers are disulfides, which are reductively cleaved by biological thiols, and peptide linkers, which are cleaved by lysosomal proteases, typically cathepsins. Significant efforts to apply disulfide linkers led to the approval and clinical testing of several agents. ${ }^{43}$ These include the first approved ADC, the acute myeloid leukemia therapy Gemtuzumab Ozogamicin (Mylotarg), which contains a hindered disulfide. ${ }^{44-46}$ However, some reports suggest that disulfides can be cleaved in circulation, leading to premature payload release and non-specific uptake. ${ }^{46-49}$ Concurrent with these efforts, cathepsin-cleavable peptide linkers have been incorporated in four approved ADCs, including the Hodgins lymphoma drug Brentuximab Vedotin (Adcetris). While these two classes of linkers have been investigated extensively, we are not aware of any reports directly comparing the relative cleavage of payloads in a solid tumor setting. To do this, we designed a small panel of probes comparing two commonly used cathepsin-cleavable peptide linkers, valine-citrulline, PanVC-CyLBam (used above) and alanine-alanine, Pan-AA-
A.

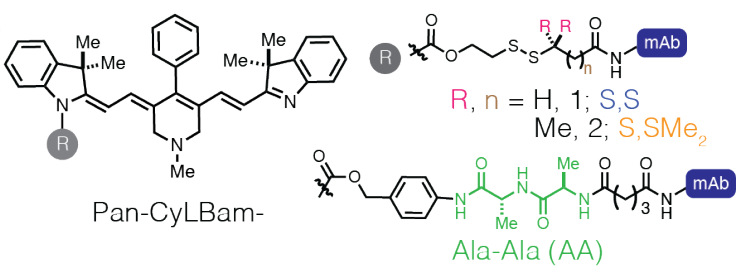

B.

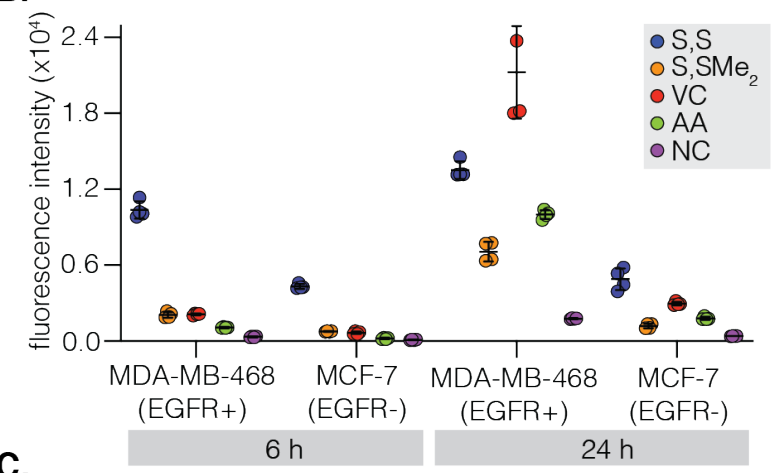

C.

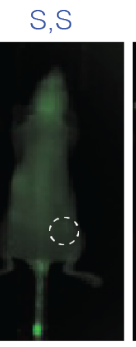

$\mathrm{S}, \mathrm{SMe}_{2}$
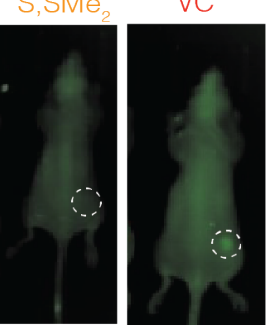

AA

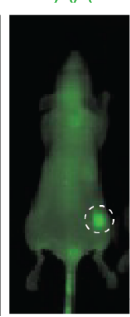

$\mathrm{NC}$

D.

E.
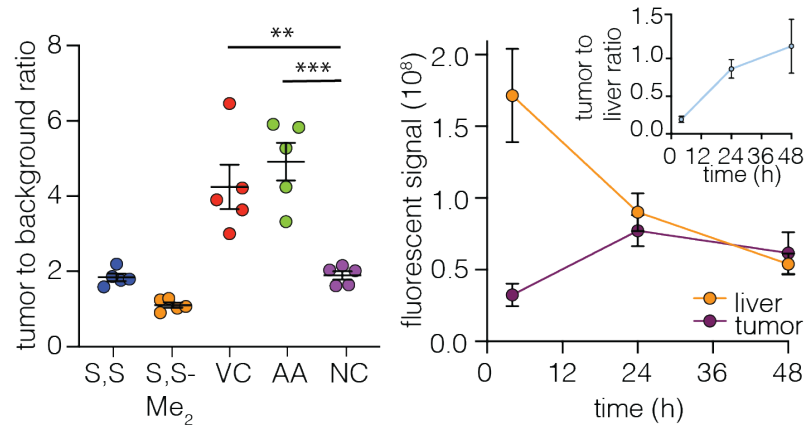

Figure 3. A. Structures of Pan-AA-CyLBam and disulfide Pan-S,S-CyLBam and Pan-S,SMe - -CyLBam. B. Quantification of in vitro uptake of the five probes $(50 \mu \mathrm{g}$; DOL 4$)$ in MDA-MB-468 (EGFR+) and MCF-7 (EGFR-) after $24 \mathrm{~h}$ incubation. Geometric mean fluorescent intensity $( \pm \mathrm{SD})$ of fluorescent signal in the cells is shown $(n=4$ independent experiments; $\sim 10,000$ cells counted). C. Fluorescent images following injection of probes $(100 \mu \mathrm{g}$; DOL 4$)$ in female athymic nude mice $(\mathrm{n}=5)$ with MDA-MB-468 tumors at $48 \mathrm{~h}$ time point. Tumors are highlighted in dotted white circles. D. Quantification of TBR 48 h post injection. E. Quantification of fluorescent signal of Pan-AA-CyLBam from liver and tumor. Tumor-to-liver ratio (TLR) is depicted in insert. Data points are displayed as mean $\pm \mathrm{SD}$, and the p-values were evaluated by the Student's t-test $(* *$ p-value $\leq 0.01$, *** pvalue $\leq 0.001)$.

CyLBam, and two disulfides, one hindered gem-dimethyl-substituted variant, Pan-S,SMe - -CyLBam and one primary disulfide, Pan-S,S-CyLBam (Figure 3A, Table S2). ${ }^{8,50}$ As a control, 
A.

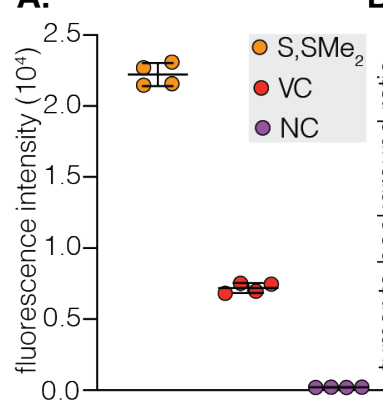

C.

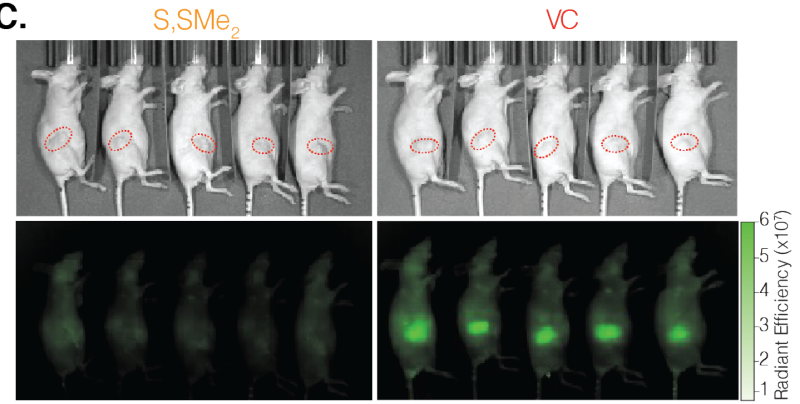

Figure 4. A. Quantification of cellular uptake of m276-SLS,SMe ${ }_{2}$-CyLBam, m276-SL-VC-CyLBam and m276-SLNC-CyLBam (80 $\mu$; DOL 4) in JIMT-1 cells following 24 $\mathrm{h}$ incubation. Geometric mean fluorescent intensity $( \pm \mathrm{SD})$ of fluorescent signal in the cells is shown $(n=4$ independent experiments; $\sim 10,000$ cells counted). B. TBRs at different time intervals after intravenous injection of m276-SLS,SMe ${ }_{2}$-CyLBam and m276-SL-VC-CyLBam $(100 \mu \mathrm{g}$; DOL 4) in female athymic nude mice bearing orthotopic JIMT-1 tumors $(n=5)$. C. Fluorescent images $72 \mathrm{~h}$ post injection. Tumors are highlighted in dotted red circles.

the non-cleavable probe, Pan-NC-CyLBam described above, was also employed.

We first examined the signal of the panel of conjugates in cells expressing variable levels of EGFR. The fluorescent signal from linker cleavage was quantified using flow cytometry at 6 and $24 \mathrm{~h}$ post-incubation (Figure 3B and S38-41). At the $6 \mathrm{~h}$ time point, Pan-S,S-CyLBam exhibite the highest fluorescent signal in EGFR+ MDA-MB-468 cells. The other three cleavable probes had only modest fluorescent signal after $6 \mathrm{~h}$, but the signal increased substantially after $24 \mathrm{~h}$. Pan-VC-CyLBam had the highest fluorescent signal among the cleavable probes after $24 \mathrm{~h}$, indicating the most extensive linker cleavage. All 5 conjugates exhibit low levels of probe signal at 6 and $24 \mathrm{~h}$ in the EGFR(-) cell lines (MCF-7 and MDA-MB-231, Figure 3B and S17, S42-43), albeit with slightly higher signal with the PanS,S-CyLBam probe. Significantly, if these data were used in isolation, it would be difficult to differentiate these linkers and assess the optimal agent for further study.

With the goal of investigating differences between these linkers in an organismal context, we then harnessed the NIR spectroscopic properties of these probes for in vivo imaging. The full series of probes $(100 \mu \mathrm{g}$ dose, half the dose of the studies above), were administered to female athymic nude mice implanted with MDA-MB-468 tumors and imaged at regular intervals up to $48 \mathrm{~h}$ (Figure 3C). With the disulfide probes, Pan$\mathbf{S}_{\mathbf{S}} \mathrm{SMe}_{2}$-CyLBam and Pan-S,S-CyLBam, we did not observe significant tumor signal, and the TBR was similar to the non- cleavable Pan-NC-CyLBam control. In contrast, strong tumor signal was observed from the cathepsin-sensitive probes, PanVC-CyLBam and Pan-AA-CyLBam (Figure 3C,D and S1921 ), with the TBR reaching as high as 4.5 after $48 \mathrm{~h}$. Therefore, these studies indicate that cathepsin-cleavable linkers provide dramatically higher tumor activation relative to hindered or non-hindered disulfides. Critically, as only modest differences are observed using the cellular methods described above, this key distinction is only apparent with in vivo imaging.

In addition to tumor uptake, these data also provide significant insight into off-target cleavage pathways. As observed above, conjugates with cleavable linkers exhibit significant liver signal at early time points (4 and $24 \mathrm{~h}$ ) (Figure S18). While likely due to a combination of liver uptake/cleavage and probe clearance through hepatobiliary pathways, this approach can provide quantitative insight into off target cleavage. With Pan-AACyLBam as an example, the liver signal decreases overtime but increases in the tumor (Figure 3E), which leads to an increase in the tumor-to-liver ratio over time (Figure 3E insert). These results are in line with prior observations suggesting significant liver linker cleavage using related cathepsin linkers. ${ }^{51-52} \mathrm{We}$ also corroborated the in vivo imaging results with an ex vivo assessment, performed $48 \mathrm{~h}$ post injection. As expected, the TMR (tumor-to-muscle ratio) revealed an identical trend to that observed in vivo (Figure S22-23).

Finally, to test the generality of our approach, CyLBams were tested against an alternative cancer target. The cell surface protein CD276, also known as B7 homolog H3 (B7H3), is overexpressed in both tumor cells and tumor vasculature of multiple solid tumor types. ${ }^{53-56}$ Antibodies against this promising target have been used to develop ADCs armed with either pyrrolobenzodiazepine (PBD) or auristatin-derived (MMAE) payloads. These ADCs, constructed using cathepsin-cleavable linkers, exhibit potent anti-tumor activity. ${ }^{57,58}$ We chose to compare VC and $\mathbf{S}, \mathbf{S M e}_{2}$ linkers and prepared the CD276 targeting antibody, m276-SL, reported previously, and non-cleavable NC control $\mathrm{m} 276-\mathrm{SL}$ conjugates, as well as a non-binding $\mathrm{mAb} \operatorname{IgG}$ controls (DOL 4, Table S4, Figure S29-30). In initial in vitro testing using a CD276+ JIMT-1 triple negative breast cancer cell line, both cleavable m276-SL conjugates showed high levels of cleavage, with little activation using the control non-binding IgG antibody (Figure 4A, and S24, S44-S45). We then carried out a study comparing protease-cleavable m276-SL-VCCyLBam and the disulfide-cleavable m276-SL-S,SMe ${ }_{2}$ CyLBam conjugates in JIMT-1 tumors $\left(200-250 \mathrm{~mm}^{3}\right)$ grown orthotopically in the mammary fat pad. As observed in the studies above, only the protease-cleavable m276-SL-VC-CyLBam conjugate exhibited a TBR greater than $5(5.2$ after $48 \mathrm{~h}$ ), while the disulfide m276-SL-S,SMe ${ }_{2}$-CyLBam probe exhibited much lower tumor signal (Figure 4B, S25-27). These initial studies in this model serve to both validate the generality of our CyBam imaging strategy, as well as the observation that protease-cleavable linkers outperform disulfide linkers in solid tumor models.

\section{CONCLUSIONS}

The mode of action of ADCs is complex - optimal efficacy depends on target binding, internalization, and, finally, lysosomal catabolism to initiate payload release. While the linker domain plays a central role in determining the efficacy and selectivity of ADCs, it is challenging to directly assess the site and extent of linker cleavage using conventional methods..$^{5-7}$ This is a 
critical issue because off-target cleavage of ADCs contributes significantly to ADC toxicity. ${ }^{59}$ The optical imaging approach detailed here provides a general means to analyze linker chemistry across cellular, tissue, and body wide scales. In this work, we identify probes suitable for $\mathrm{mAb}$-targeted activatable imaging. While our previously reported sulfonated $\mathbf{C y B a m s}$ were not suitable for this application, installation of a tertiary amine into the norcyanine framework dramatically improves cellular and in vivo signal, likely due to enhanced lysosomal uptake and retention. It is likely that these "lysotracker-like" norcyanines and the resulting CyLBam probes will have applications in other settings, including their use as activity-based sensing agents. $^{60-61}$

In applying this approach for ADC applications, we assume that the fluorescent probe can serve as a useful surrogate for the payload component. Here we have shown that quantitative comparisons of linker chemistries can be obtained by varying the linker component. In addition to linker chemistry, the properties of the payload molecule (e.g. charge, hydrophobicity) are also important aspects of tumor targeting. ${ }^{62-65}$ Critically, while the norcyanine probe is a hydrophobic, $\mathrm{sp}^{2}$-rich small molecule, so too are many ADC payloads. ${ }^{66-69}$ Further variations of the cyanine component could provide additional insights into the role of payload properties on tumor and normal tissue distributions, something our group has examined previously in the context of always-ON fluorophore conjugates. ${ }^{40,70-73}$

Going forward, we anticipate that CyLBam imaging could provide insights that directly inform the development of ADCs. These studies have suggested significant differences between protease cleavable dipeptides and disulfides in terms of tumor cleavage, which is likely due to instability of disulfides in circulation. Prior studies looking at various cysteine mutants have found the site of $\mathrm{mAb}$ labeling can have dramatic effects on disulfide stability. ${ }^{74}$ This approach is well positioned to investigate these and related homogenous labeling strategies. We also anticipate this strategy can readily be extended to analyze the impact of $\mathrm{mAb}$ engineering strategies. ${ }^{75-77}$ As altering the molecular weight and composition of the protein component can dramatically alter the clearance pathway, it is likely that linker cleavage dynamics will be impacted. We also note this strategy can readily be translated to other targeted drug delivery strategies that rely on cleavable linkers. Efforts towards these goals, and to translate lessons from these imaging efforts into novel therapeutics, are underway.

\section{ASSOCIATED CONTENT}

\section{Supporting Information}

This material is available free of charge via the internet at http://pubs.acs.org. Supporting Information contain synthesis procedure and characterization of NorCy7s, CyBams, CyLBams, and key intermediate compounds, photophysical properties of NorCy7 analogs and CyLBams, details of in vitro experiments and supporting figures and tables.

\section{AUTHOR INFORMATION}

\section{Corresponding Author}

* Email: martin.schnermann@nih.gov

\section{Author Contributions}

The manuscript was written through contributions of all authors. All authors have given approval to the final version of the manuscript.

\section{ABBREVIATIONS}

NIR, near-infrared; ADC, Antibody Drug Conjugate; mAb, monoclonal antibody; CyBam, cyanine carbamate; CyLBam, cyanine lysosomal carbamate; ELISA, enzyme-linked immunosorbent assay,; PBS, phosphate buffer saline; B7H3, B7 homo$\log \mathrm{H} 3$; IVIS, in vivo imaging system; CD276, cluster of differentiation 276; EGFR, epidermal growth factor; abs, absorbance; ex, excitation; em, emission; N.A, numerical aperture; VC, valine-citrulline, AA, alanine-alanine, NC, non-cleavable; Pan, panitumumab; NHS, $N$-hydroxy succinimide; DOL, degree of labeling; TBR, tumor-to-background ratio; TLR, liver-to-liver ratio; TMR, tumor-to-muscle ratio; IgG, immunoglobulin $\mathrm{G}$; $\mathrm{MeOH}$, methanol; SD, standard deviation.

\section{ACKNOWLEDGMENT}

This work was supported by the Intramural Research Program of the National Institutes of Health (NIH), NCI-CCR. It was also supported by an NCI CCR FLEX Program Synergy Award (to M.S. and B.S.C.) and a Congressionally Directed Medical Research Program (CDRMP) Breast Cancer Research Program grant (award number W81WXH21-1-0109). We acknowledge Dr. James A. Kelley (National Cancer Institute) for providing the high-resolution mass spectrometry analysis. We thank Dr. Gary T. Pauli (National Cancer Institute) for assisting with LC/MS and HPLC purification. The Biophysics Resource, CCR is acknowledged for use of instrumentation. We would also like to thank Dr. Valentin Magidson, NCI-Optical Microscopy laboratory, and Dr. Jeff Carrell (CCRFrederick Flow Cytometry Core Laboratory) for assisting with confocal microscopy and flow cytometry respectively.

\section{REFERENCES}

1. Beck, A.; Goetsch, L.; Dumontet, C.; Corvaia, N., Strategies and challenges for the next generation of antibody-drug conjugates. Nat Rev Drug Discov 2017, 16 (5), 315-337.

2. Chau, C. H.; Steeg, P. S.; Figg, W. D., Antibody-drug conjugates for cancer. Lancet 2019, 394 (10200), 793-804.

3. Drago, J. Z.; Modi, S.; Chandarlapaty, S., Unlocking the potential of antibody-drug conjugates for cancer therapy. Nat Rev Clin Oncol 2021, 18 (6), 327-344.

4. Kopp, A.; Thurber, G. M., Severing Ties: Quantifying the Payload Release from Antibody Drug Conjugates. Cell Chem Biol 2019, 26 (12), 1631-1633.

5. Su, D.; Zhang, D., Linker Design Impacts Antibody-Drug Conjugate Pharmacokinetics and Efficacy via Modulating the Stability and Payload Release Efficiency. Front Pharmacol 2021, 12, 687926.

6. Dal Corso, A.; Pignataro, L.; Belvisi, L.; Gennari, C., Innovative Linker Strategies for Tumor-Targeted Drug Conjugates. Chemistry 2019, 25 (65), 14740-14757.

7. Tsuchikama, K.; An, Z., Antibody-drug conjugates: recent advances in conjugation and linker chemistries. Protein Cell 2018, 9 (1), 33-46.

8. Lewis Phillips, G. D.; Li, G.; Dugger, D. L.; Crocker, L. M.; Parsons, K. L.; Mai, E.; Blattler, W. A.; Lambert, J. M.; Chari, R. V.; Lutz, R. J.; Wong, W. L.; Jacobson, F. S.; Koeppen, H.; Schwall, R. H.; Kenkare-Mitra, S. R.; Spencer, S. D.; Sliwkowski, M. X., Targeting 
HER2-positive breast cancer with trastuzumab-DM1, an antibodycytotoxic drug conjugate. Cancer Res 2008, 68 (22), 9280-90.

9. Sun, X.; Widdison, W.; Mayo, M.; Wilhelm, S.; Leece, B.; Chari, R.; Singh, R.; Erickson, H., Design of antibody-maytansinoid conjugates allows for efficient detoxification via liver metabolism. Bioconjug Chem 2011, 22 (4), 728-35.

10. Kovtun, Y. V.; Audette, C. A.; Ye, Y.; Xie, H.; Ruberti, M. F.; Phinney, S. J.; Leece, B. A.; Chittenden, T.; Blattler, W. A.; Goldmacher, V. S., Antibody-drug conjugates designed to eradicate tumors with homogeneous and heterogeneous expression of the target antigen. Cancer Res 2006, 66 (6), 3214-21.

11. Chang, H. P.; Shah, D. K., Determination of ADC Concentration by Ligand-Binding Assays. Methods Mol Biol 2020, 2078, 361-369.

12. Cahuzac, H.; Devel, L., Analytical Methods for the Detection and Quantification of ADCs in Biological Matrices. Pharmaceuticals (Basel) 2020, 13 (12).

13. Garousi, J.; Orlova, A.; Frejd, F. Y.; Tolmachev, V., Imaging using radiolabelled targeted proteins: radioimmunodetection and beyond. EJNMMI Radiopharm Chem 2020, 5 (1), 16.

14. Allen, K. J. H.; Jiao, R.; Malo, M. E.; Frank, C.; Dadachova, E., Biodistribution of a Radiolabeled Antibody in Mice as an Approach to Evaluating Antibody Pharmacokinetics. Pharmaceutics 2018, 10 (4).

15. Fawwaz, R. A.; Oluwole, S.; Srivastava, S.; Iga, C.; Wang, T.; Rosen, J. M.; Hardy, M. A.; Alderson, P. O., The Biodistribution of Radiolabeled Antilymphocyte Monoclonal-Antibody in the Rat. $\mathrm{Nucl}$ Med Biol 1986, 13 (1), 39-42.

16. Westrom, S.; Bonsdorff, T. B.; Abbas, N.; Bruland, O. S.; Jonasdottir, T. J.; Maelandsmo, G. M.; Larsen, R. H., Evaluation of CD146 as Target for Radioimmunotherapy against Osteosarcoma. Plos One 2016, 11 (10), e0165382.

17. Sorkin, M. R.; Walker, J. A.; Kabaria, S. R.; Torosian, N. P.; Alabi, C. A., Responsive Antibody Conjugates Enable Quantitative Determination of Intracellular Bond Degradation Rate. Cell Chem Biol 2019, 26 (12), 1643-1651 e4.

18. Lee, B. C.; Chalouni, C.; Doll, S.; Nalle, S. C.; Darwish, M.; Tsai, S. P.; Kozak, K. R.; Del-Rosario, G.; Yu, S. F.; Erickson, H.; Vandlen, R., FRET Reagent Reveals the Intracellular Processing of PeptideLinked Antibody-Drug Conjugates. Bioconjug Chem 2018, 29 (7), 2468-2477.

19. Knewtson, K. E.; Perera, C.; Hymel, D.; Gao, Z.; Lee, M. M.; Peterson, B. R., Antibody-Drug Conjugate that Exhibits Synergistic Cytotoxicity with an Endosome-Disruptive Peptide. ACS Omega 2019, 4 (7), 12955-12968.

20. Cilliers, C.; Nessler, I.; Christodolu, N.; Thurber, G. M., Tracking Antibody Distribution with Near-Infrared Fluorescent Dyes: Impact of Dye Structure and Degree of Labeling on Plasma Clearance. Mol Pharm 2017, 14 (5), 1623-1633.

21. Lu, G.; Nishio, N.; van den Berg, N. S.; Martin, B. A.; Fakurnejad, S.; van Keulen, S.; Colevas, A. D.; Thurber, G. M.; Rosenthal, E. L., Co-administered antibody improves penetration of antibody-dye conjugate into human cancers with implications for antibody-drug conjugates. Nat Commun 2020, 11 (1), 5667.

22. Poplinger, D.; Bokan, M.; Hesin, A.; Thankarajan, E.; Tuchinsky, H.; Gellerman, G.; Patsenker, L., Ratiometric Fluorescence Monitoring of Antibody-Guided Drug Delivery to Cancer Cells. Bioconjug Chem 2021.

23. Thankarajan, E.; Jadhav, S.; Luboshits, G.; Gellerman, G.; Patsenker, L., Quantification of Drug Release Degree In Vivo Using Antibody-Guided, Dual-NIR-Dye Ratiometric System. Anal Chem 2021, 93 (23), 8265-8272.

24. Usama, S. M.; Inagaki, F.; Kobayashi, H.; Schnermann, M. J., Norcyanine-Carbamates Are Versatile Near-Infrared Fluorogenic Probes. J Am Chem Soc 2021, 143 (15), 5674-5679.

25. Keppler, A.; Arrivoli, C.; Sironi, L.; Ellenberg, J., Fluorophores for live cell imaging of AGT fusion proteins across the visible spectrum. Biotechniques 2006, 41 (2), 167-70, 172, 174-5.

26. Caldwell, S. T.; O'Byrne, S. N.; Wilson, C.; Cvetko, F.; Murphy, M. P.; McCarron, J. G.; Hartley, R. C., Photoactivated release of membrane impermeant sulfonates inside cells. Chem Commun (Camb) 2021, 57 (32), 3917-3920.

27. Yang, N. J.; Hinner, M. J., Getting across the cell membrane: an overview for small molecules, peptides, and proteins. Methods Mol Biol 2015, 1266, 29-53.

28. Yang, Z.; Usama, S. M.; Li, F.; Burgess, K.; Li, Z., A zwitterionic near-infrared dye linked TrkC targeting agent for imaging metastatic breast cancer. Medchemcomm 2018, 9 (10), 1754-1760.

29. Hyun, H.; Owens, E. A.; Narayana, L.; Wada, H.; Gravier, J.; Bao, K.; Frangioni, J. V.; Choi, H. S.; Henary, M., Central C-C Bonding Increases Optical and Chemical Stability of NIR Fluorophores. Rsc Adv 2014, 4 (102), 58762-58768.

30. Yang, C.; Wang, H.; Yokomizo, S.; Hickey, M.; Chang, H.; Kang, H.; Fukuda, T.; Song, M. Y.; Lee, S. Y.; Park, J. W.; Bao, K.; Choi, H. S., ZW800-PEG: A Renal Clearable Zwitterionic Near-Infrared Fluorophore for Potential Clinical Translation. Angew Chem Int Ed Engl 2021, 60 (25), 13847-13852.

31. Lee, H.; Mason, J. C.; Achilefu, S., Heptamethine cyanine dyes with a robust $\mathrm{C}-\mathrm{C}$ bond at the central position of the chromophore. J Org Chem 2006, 71 (20), 7862-5.

32. Samanta, A.; Vendrell, M.; Das, R.; Chang, Y. T., Development of photostable near-infrared cyanine dyes. Chem Commun (Camb) 2010, 46 (39), 7406-8.

33. Usama, S. M.; Burgess, K., Hows and Whys of Tumor-Seeking Dyes. Acc Chem Res 2021, 54 (9), 2121-2131.

34. Xu, W.; Zeng, Z.; Jiang, J. H.; Chang, Y. T.; Yuan, L., Discerning the Chemistry in Individual Organelles with Small-Molecule Fluorescent Probes. Angew Chem Int Ed Engl 2016, 55 (44), 1365813699.

35. Zhu, H.; Fan, J.; Du, J.; Peng, X., Fluorescent Probes for Sensing and Imaging within Specific Cellular Organelles. Acc Chem Res 2016, 49 (10), 2115-2126.

36. Choi, N. E.; Lee, J. Y.; Park, E. C.; Lee, J. H.; Lee, J., Recent Advances in Organelle-Targeted Fluorescent Probes. Molecules 2021, 26 (1).

37. Gorka, A. P.; Nani, R. R.; Schnermann, M. J., Harnessing Cyanine Reactivity for Optical Imaging and Drug Delivery. Acc Chem Res 2018, 51 (12), 3226-3235.

38. Cilliers, C.; Menezes, B.; Nessler, I.; Linderman, J.; Thurber, G. M., Improved Tumor Penetration and Single-Cell Targeting of Antibody-Drug Conjugates Increases Anticancer Efficacy and Host Survival. Cancer Res 2018, 78 (3), 758-768.

39. Cilliers, C.; Liao, J.; Atangcho, L.; Thurber, G. M., Residualization Rates of Near-Infrared Dyes for the Rational Design of Molecular Imaging Agents. Mol Imaging Biol 2015, 17 (6), 757-62.

40. Usama, S. M.; Thapaliya, E. R.; Luciano, M. P.; Schnermann, M. J., Not so innocent: Impact of fluorophore chemistry on the in vivo properties of bioconjugates. Curr Opin Chem Biol 2021, 63, 38-45.

41. Mamot, C.; Drummond, D. C.; Greiser, U.; Hong, K.; Kirpotin, D. B.; Marks, J. D.; Park, J. W., Epidermal growth factor receptor (EGFR)-targeted immunoliposomes mediate specific and efficient drug delivery to EGFR- and EGFRvIII-overexpressing tumor cells. Cancer Res 2003, 63 (12), 3154-61.

42. Bhattacharyya, S.; Patel, N.; Wei, L.; Riffle, L. A.; Kalen, J. D.; Hill, G. C.; Jacobs, P. M.; Zinn, K. R.; Rosenthal, E., Synthesis and biological evaluation of panitumumab-IRDye800 conjugate as a fluorescence imaging probe for EGFR-expressing cancers. Medchemcomm 2014, 5 (9), 1337-1346.

43. Wang, Q.; Guan, J. K.; Wan, J. L.; Li, Z. F., Disulfide based prodrugs for cancer therapy. Rsc Adv 2020, 10 (41), 24397-24409.

44. Widdison, W. C.; Wilhelm, S. D.; Cavanagh, E. E.; Whiteman, K. R.; Leece, B. A.; Kovtun, Y.; Goldmacher, V. S.; Xie, H.; Steeves, R. M.; Lutz, R. J.; Zhao, R.; Wang, L.; Blattler, W. A.; Chari, R. V., Semisynthetic maytansine analogues for the targeted treatment of cancer. $J$ Med Chem 2006, 49 (14), 4392-408.

45. Liu, C.; Tadayoni, B. M.; Bourret, L. A.; Mattocks, K. M.; Derr, S. M.; Widdison, W. C.; Kedersha, N. L.; Ariniello, P. D.; Goldmacher, V. S.; Lambert, J. M.; Blattler, W. A.; Chari, R. V., Eradication of large 
colon tumor xenografts by targeted delivery of maytansinoids. Proc Natl Acad Sci U S A 1996, 93 (16), 8618-23.

46. Kellogg, B. A.; Garrett, L.; Kovtun, Y.; Lai, K. C.; Leece, B.; Miller, M.; Payne, G.; Steeves, R.; Whiteman, K. R.; Widdison, W.; Xie, H.; Singh, R.; Chari, R. V.; Lambert, J. M.; Lutz, R. J., Disulfide-linked antibody-maytansinoid conjugates: optimization of in vivo activity by varying the steric hindrance at carbon atoms adjacent to the disulfide linkage. Bioconjug Chem 2011, 22 (4), 717-27.

47. Danial, M.; Postma, A., Disulfide conjugation chemistry: a mixed blessing for therapeutic drug delivery? Ther Deliv 2017, 8 (6), 359-362.

48. Fourie-O'Donohue, A.; Chu, P. Y.; Dela Cruz Chuh, J.; Tchelepi, R.; Tsai, S. P.; Tran, J. C.; Sawyer, W. S.; Su, D.; Ng, C.; Xu, K.; Yu, S. F.; Pillow, T. H.; Sadowsky, J.; Dragovich, P. S.; Liu, Y.; Kozak, K. R., Improved translation of stability for conjugated antibodies using an in vitro whole blood assay. MAbs 2020, 12 (1), 1715705.

49. Xie, H.; Audette, C.; Hoffee, M.; Lambert, J. M.; Blattler, W. A., Pharmacokinetics and biodistribution of the antitumor immunoconjugate, cantuzumab mertansine (huC242-DM1), and its two components in mice. J Pharmacol Exp Ther 2004, 308 (3), 1073-82.

50. Salomon, P. L.; Reid, E. E.; Archer, K. E.; Harris, L.; Maloney, E. K.; Wilhelm, A. J.; Miller, M. L.; Chari, R. V. J.; Keating, T. A.; Singh, R., Optimizing Lysosomal Activation of Antibody-Drug Conjugates (ADCs) by Incorporation of Novel Cleavable Dipeptide Linkers. Mol Pharm 2019, 16 (12), 4817-4825.

51. Dubowchik, G. M.; Firestone, R. A.; Padilla, L.; Willner, D.; Hofstead, S. J.; Mosure, K.; Knipe, J. O.; Lasch, S. J.; Trail, P. A., Cathepsin B-labile dipeptide linkers for lysosomal release of doxorubicin from internalizing immunoconjugates: model studies of enzymatic drug release and antigen-specific in vitro anticancer activity. Bioconjug Chem 2002, 13 (4), 855-69.

52. Caculitan, N. G.; Dela Cruz Chuh, J.; Ma, Y.; Zhang, D.; Kozak, K. R.; Liu, Y.; Pillow, T. H.; Sadowsky, J.; Cheung, T. K.; Phung, Q.; Haley, B.; Lee, B. C.; Akita, R. W.; Sliwkowski, M. X.; Polson, A. G., Cathepsin B Is Dispensable for Cellular Processing of Cathepsin BCleavable Antibody-Drug Conjugates. Cancer Res 2017, 77 (24), 7027-7037.

53. Qin, X.; Zhang, H.; Ye, D.; Dai, B.; Zhu, Y.; Shi, G., B7-H3 is a new cancer-specific endothelial marker in clear cell renal cell carcinoma. Onco Targets Ther 2013, 6, 1667-73.

54. Zang, X.; Sullivan, P. S.; Soslow, R. A.; Waitz, R.; Reuter, V. E.; Wilton, A.; Thaler, H. T.; Arul, M.; Slovin, S. F.; Wei, J.; Spriggs, D. R.; Dupont, J.; Allison, J. P., Tumor associated endothelial expression of B7-H3 predicts survival in ovarian carcinomas. Mod Pathol 2010, 23 (8), 1104-12.

55. Brunner, A.; Hinterholzer, S.; Riss, P.; Heinze, G.; Brustmann, H., Immunoexpression of B7-H3 in endometrial cancer: relation to tumor T-cell infiltration and prognosis. Gynecol Oncol 2012, 124 (1), 105-11.

56. Seaman, S.; Stevens, J.; Yang, M. Y.; Logsdon, D.; Graff-Cherry, C.; St Croix, B., Genes that distinguish physiological and pathological angiogenesis. Cancer Cell 2007, 11 (6), 539-54.

57. Seaman, S.; Zhu, Z.; Saha, S.; Zhang, X. M.; Yang, M. Y.; Hilton, M. B.; Morris, K.; Szot, C.; Morris, H.; Swing, D. A.; Tessarollo, L.; Smith, S. W.; Degrado, S.; Borkin, D.; Jain, N.; Scheiermann, J.; Feng, Y.; Wang, Y.; Li, J.; Welsch, D.; DeCrescenzo, G.; Chaudhary, A.; Zudaire, E.; Klarmann, K. D.; Keller, J. R.; Dimitrov, D. S.; St Croix, B., Eradication of Tumors through Simultaneous Ablation of CD276/B7-H3-Positive Tumor Cells and Tumor Vasculature. Cancer Cell 2017, 31 (4), 501-515 e8.

58. Kendsersky, N. M.; Lindsay, J.; Kolb, E. A.; Smith, M. A.; Teicher, B. A.; Erickson, S. W.; Earley, E. J.; Mosse, Y. P.; Martinez, D.; Pogoriler, J.; Krytska, K.; Patel, K.; Groff, D.; Tsang, M.; Ghilu, S.; Wang, Y. F.; Seaman, S.; Feng, Y.; St Croix, B.; Gorlick, R.; Kurmasheva, R.; Houghton, P. J.; Maris, J. M., The B7-H3-Targeting Antibody-Drug Conjugate m276-SL-PBD Is Potently Effective Against Pediatric Cancer Preclinical Solid Tumor Models. Clin Cancer Res 2021, 27 (10), 2938-2946.
59. Masters, J. C.; Nickens, D. J.; Xuan, D.; Shazer, R. L.; Amantea, M., Clinical toxicity of antibody drug conjugates: a meta-analysis of payloads. Invest New Drugs 2017.

60. Bruemmer, K. J.; Crossley, S. W. M.; Chang, C. J., Activity-Based Sensing: A Synthetic Methods Approach for Selective Molecular Imaging and Beyond. Angewandte Chemie 2020, 59 (33), 13734 13762.

61. Scott, J. I.; Deng, Q.; Vendrell, M., Near-Infrared Fluorescent Probes for the Detection of Cancer-Associated Proteases. Acs Chem Biol 2021, 16 (8), 1304-1317.

62. Shao, T.; Chen, T.; Chen, Y.; Liu, X.; Chen, Y. L.; Wang, Q.; Zhu, T.; Guo, M.; Li, H.; Ju, D.; Wang, C., Construction of paclitaxel-based antibody-drug conjugates with a PEGylated linker to achieve superior therapeutic index. Signal Transduct Target Ther 2020, 5 (1), 132.

63. Buecheler, J. W.; Winzer, M.; Tonillo, J.; Weber, C.; Gieseler, H., Impact of Payload Hydrophobicity on the Stability of AntibodyDrug Conjugates. Mol Pharm 2018, 15 (7), 2656-2664.

64. Singh, S. K.; Luisi, D. L.; Pak, R. H., Antibody-Drug Conjugates: Design, Formulation and Physicochemical Stability. Pharm Res 2015, 32 (11), 3541-71.

65. Hamblett, K. J.; Senter, P. D.; Chace, D. F.; Sun, M. M.; Lenox, J.; Cerveny, C. G.; Kissler, K. M.; Bernhardt, S. X.; Kopcha, A. K.; Zabinski, R. F.; Meyer, D. L.; Francisco, J. A., Effects of drug loading on the antitumor activity of a monoclonal antibody drug conjugate. Clin Cancer Res 2004, 10 (20), 7063-70.

66. Hollander, I.; Kunz, A.; Hamann, P. R., Selection of reaction additives used in the preparation of monomeric antibody-calicheamicin conjugates. Bioconjug Chem 2008, 19 (1), 358-61.

67. Zhao, R. Y.; Wilhelm, S. D.; Audette, C.; Jones, G.; Leece, B. A.; Lazar, A. C.; Goldmacher, V. S.; Singh, R.; Kovtun, Y.; Widdison, W. C.; Lambert, J. M.; Chari, R. V., Synthesis and evaluation of hydrophilic linkers for antibody-maytansinoid conjugates. J Med Chem 2011, 54 (10), 3606-23.

68. Christie, R. J.; Tiberghien, A. C.; Du, Q.; Bezabeh, B.; Fleming, R.; Shannon, A.; Mao, S.; Breen, S.; Zhang, J.; Zhong, H.; Harper, J.; Wu, H.; Howard, P. W.; Gao, C., Pyrrolobenzodiazepine AntibodyDrug Conjugates Designed for Stable Thiol Conjugation. Antibodies (Basel) 2017, 6 (4).

69. Mendelsohn, B. A.; Barnscher, S. D.; Snyder, J. T.; An, Z.; Dodd, J. M.; Dugal-Tessier, J., Investigation of Hydrophilic Auristatin Derivatives for Use in Antibody Drug Conjugates. Bioconjug Chem 2017, 28 (2), 371-381.

70. Luciano, M. P.; Crooke, S. N.; Nourian, S.; Dingle, I.; Nani, R. R.; Kline, G.; Patel, N. L.; Robinson, C. M.; Difilippantonio, S.; Kalen, J. D.; Finn, M. G.; Schnermann, M. J., A Nonaggregating Heptamethine Cyanine for Building Brighter Labeled Biomolecules. Acs Chem Biol 2019, 14 (5), 934-940.

71. Sato, K.; Gorka, A. P.; Nagaya, T.; Michie, M. S.; Nakamura, Y.; Nani, R. R.; Coble, V. L.; Vasalatiy, O. V.; Swenson, R. E.; Choyke, P. L.; Schnermann, M. J.; Kobayashi, H., Effect of charge localization on the in vivo optical imaging properties of near-infrared cyanine dye/monoclonal antibody conjugates. Mol Biosyst 2016, 12 (10), 3046-56.

72. Sato, K.; Nagaya, T.; Nakamura, Y.; Harada, T.; Nani, R. R.; Shaum, J. B.; Gorka, A. P.; Kim, I.; Paik, C. H.; Choyke, P. L.; Schnermann, M. J.; Kobayashi, H., Impact of C4'-O-Alkyl Linker on in Vivo Pharmacokinetics of Near-Infrared Cyanine/Monoclonal Antibody Conjugates. Mol Pharm 2015, 12 (9), 3303-11.

73. Cha, J.; Nani, R. R.; Luciano, M. P.; Kline, G.; Broch, A.; Kim, K.; Namgoong, J. M.; Kulkarni, R. A.; Meier, J. L.; Kim, P.; Schnermann, M. J., A chemically stable fluorescent marker of the ureter. Bioorg Med Chem Lett 2018, 28 (16), 2741-2745.

74. Ohri, R.; Bhakta, S.; Fourie-O'Donohue, A.; Dela Cruz-Chuh, J.; Tsai, S. P.; Cook, R.; Wei, B.; Ng, C.; Wong, A. W.; Bos, A. B.; Farahi, F.; Bhakta, J.; Pillow, T. H.; Raab, H.; Vandlen, R.; Polakis, P.; Liu, Y.; Erickson, H.; Junutula, J. R.; Kozak, K. R., High-Throughput Cysteine Scanning To Identify Stable Antibody Conjugation Sites for 
Maleimide- and Disulfide-Based Linkers. Bioconjug Chem 2018, 29 (2), 473-485.

75. Dorywalska, M.; Strop, P.; Melton-Witt, J. A.; Hasa-Moreno, A.; Farias, S. E.; Casas, M. G.; Delaria, K.; Lui, V.; Poulsen, K.; Sutton, J.; Bolton, G.; Zhou, D. H.; Moine, L.; Dushin, R.; Tran, T. T.; Liu, S. H.; Rickert, M.; Foletti, D.; Shelton, D. L.; Pons, J.; Rajpal, A., Site-Dependent Degradation of a Non-Cleavable Auristatin-Based LinkerPayload in Rodent Plasma and Its Effect on ADC Efficacy. Plos One 2015, 10 (7).
76. Liu, L., Pharmacokinetics of monoclonal antibodies and Fcfusion proteins. Protein Cell 2018, 9 (1), 15-32.

77. Kelly, R. L.; Yu, Y.; Sun, T.; Caffry, I.; Lynaugh, H.; Brown, M.; Jain, T.; Xu, Y.; Wittrup, K. D., Target-independent variable region mediated effects on antibody clearance can be FcRn independent. MAbs 2016, 8 (7), 1269-1275. 